\title{
The network is not the territory: On capturing mobile media
}

new media \& society 2019, Vol. 2I(I) 77-96 (C) The Author(s) 2018

Article reuse guidelines: sagepub.com/journals-permissions DOI: |0.I |77/|46|4448|878735 | journals.sagepub.com/home/nms

@SAGE

\section{Florian Sprenger}

Goethe University Frankfurt, Germany

\begin{abstract}
Cellular mobile networks, in which devices constantly relay their location and their movements, are formed by the motion of end devices in relation to the position of radio towers. As a matter of principle, it is this motion that allows the location of devices to be identified within the network. The article argues that the emergence of mobile media based on cellular triangulation has introduced an ontology in which, by technical necessity, the position of every object is constantly registered and objects that do not have an address do not exist. The location and movement of all participants are, at all times, a known technical variable. With Xeros PARC's "ubiquitous computing" as a reference case, the article scrutinizes how movement triggers the process that registers the locations of mobile phones or smartphones, a development it situates against the cybernetic imagination of determining the location and the movement of an object at the same time.
\end{abstract}

\section{Keywords}

Addressability, capture, cellular triangulation, cybernetics, digital cultures, German media theory, media archeology, mobile media, Philip Agre

The whole history of cybernetics has aimed to do away with the impossibility of determining at the same time the position and behavior of bodies.-Tiqqun, "The Cybernetic Hypothesis"

With these words, the collective known as Tiqqun (2010 [2001]) described the history of cybernetics as a series of technical attempts to automatize predictions about the behavior

\section{Corresponding author:}

Florian Sprenger, Goethe University Frankfurt, Institut für Theater-, Film- und Medienwissenschaft, Norbert-Wollheim-Platz I, 60323 Frankfurt am Main, Germany.

Email: sprenger@tfm.uni-frankfurt.de 
and motion of objects based upon probabilities. If the object whose movement or behavior is to be observed happens to move, then it will already be in another location in the time it takes to react and transmit information about this very movement to the observer. The decisive term in this quote is "at the same time": position and behavior can be determined separately and in succession, but to observe them simultaneously is a physical impossibility. In this regard, the development of cybernetic technologies is entwined with physical-philosophical problems related to media of transmission: How can something act at two places at the same time? And how can movement be determined and predicted from a distance, when transmission over this distance takes time?

Following Tiqqun's intuition, such questions of mediation can be found at the epistemological core of cybernetics. It is not possible to determine the movement and the position of a body at the same time because the observer is located in time and space. There is no instantaneous medium of immediate simultaneity. The quote by Tiqqun suggests that the history of cybernetics can be understood as a search for-or rather imagination of - other technical solutions to the challenge of relativity.

In this context, one of the most sophisticated solution strategies of the early $21 \mathrm{st}$ century consists in technological objects that constantly track and register their own position and behavior. In mobile cellular networks, which spread over almost the whole planet in the past 20 years, every cellular device is constantly localized. Localization, as it will turn out, is a technological prerequisite of connection to such networks. This article describes how technologies of localization based on the principles of cellular triangulation - the determination of a device's position within the network of transmitters and other devices - developed as an answer to the challenges of relativity by introducing new modes of addressing. Consequently, mobile technologies of addressability, as they are deeply embedded into daily routines today, turn out as the latest iteration of the cybernetic imaginary described by Tiqqun.

Although Tiqqun does not discuss specific technologies, the history of cybernetics reveals that the quote from the beginning refers to one of cybernetic's origins, as described by Peter Galison (1994): the anti-aircraft predictors that Norbert Wiener researched during World War II. In the time needed for a bullet or a missile to reach its flying target, the target will already be elsewhere. Thus in order to strike a moving airplane, the shot has to be directed at the plane's future location, and this cannot be done without making extrapolations about its flight path and without introducing a feedback loop between the speed of both the target and the missile. The missile is always aimed at the future, where its target is most likely to be. Controlling and coordinating attacks to strike the future positions of an object requires technologies that overcome or avoid the relativity associated with every transmission. The operational and epistemic impossibility of accomplishing this lies in simultaneously determining both of these variableslocation and motion - in a single act of observation. It follows that the information processed by cybernetic machines about the objects under their control necessarily contains a gap between the past and the future. They never know the present.

On the basis of this gap of relativity, Tiqqun's intuition about the cybernetic imagination can be applied to the various histories of technical solutions devised in the 20th century. Beginning with the anti-aircraft predictor, technologies that require constant transmission such as missiles, spacecraft, or drones relied on both improved predictions 
about the behavior of objects and the reduction of contingency. The basic approach is to predict the behavior of the observed bodies in order to determine their future positions, because the previous movement of an object does not allow to unambiguously deduce its continuation. Such solutions include the use of feedback loops to extrapolate the future locations of objects, the invention of objects that can independently correct their path by registering their own movement, and finally the self-registration of movements - in other words: from the anti-aircraft predictor over cruise missiles that update their target during flight to drones whose movements can be tracked and operated from a distance.

The cellular technologies discussed here obviously differ from these technologies. While the missile or the automated drone are determined to reach specific coordinates at a specific time, it is characteristic for mobile devices (respectively, their users) that they can move (almost) everywhere without losing connectivity. The coverage of radio towers is determined by their electromagnetic field. Technologies of cellular triangulation nonetheless employ time-critical transmissions that make it possible to localize devices while they are moving. In fact, in the act of movement the data used to locate the device are retrieved. In this sense, such technologies react to the same challenge as missiles or drones and can be described as a part of a new solution: technologies of automatic selfregistration rely on principles of capture, summarized in 1994 by Philip Agre (1994), in which the activity being monitored generates monitored data as it is undertaken. Early examples are customer cards which create a profile while shopping, the economy of social media, in which new connections account for graphs, and fitness-trackers which register health status while doing sports. Referring to spatial processes, technologies of capture allow the tracking of an object's movements and consequently to observe position and behavior supposedly at the same time.

Technologies of capture are ubiquitous in daily life with mobile media: cellular devices employ them on different levels to determine their own position. Their networks of mobility, based on the principles of cellular triangulation and technologies of addressing, attempt to conclude the challenge of the relativity of movement and its observation by introducing an ontology in which existence equals addressability. By technical necessity, the position of every object is constantly registered and objects that do not have an address do not exist. The solution implemented in such technologies is to make objects relay their movement and to transform this information into a network in which the position of every object is constantly traced and tracked. Such networks, for which the surrounding cellular networks with their advanced addressing system are the dominant example, consist of relations between objects, whose position and movement are registered, rather than of coordinates in geographical space. This difference between network and territory is important to understand how such technologies couple space and movement in a new way.

To understand what is at stake with this development, it is important to put the term address into the center of attention. Addresses foster the allocation of devices and positions in the network. In order for a transmission to take place between one location and another, these locations need addresses. It is necessary to know, in other words, where the information about an object's presence is transmitted from and where it is transmitted to. In this sense, addresses allow for correlations to be made between particular positions and particular data. As uniformly stored data sets, addresses can move along with objects 
in space, mark the positions where they can be reached and thus have effects on surrounding spaces. As an element of transmission processes, addressability is bound to the relativistic challenge.

In this sense, networked mobile devices with assigned addresses are constantly documenting their presence or absence in certain locations. Necessarily, and as a matter of principle, it is addressability that allows the location of devices to be identified within the network and makes their users reachable during movement: their devices can be addressed to transmit and to receive data even if they change their position. As a technology without interruptions, the mobile cellular network creates reachability by enabling constant reception. Reachability implies seamless connectivity by addressability at least as far as infrastructures reach. To be addressable is thus a technical mode of mobile existence; that which cannot be addressed does not exist within networks of transmission because it has no location and cannot move.

A world in which media are mobile in this way-in which we can move freely and our cellular devices register our location - is thus a world in which the location and movement of all participants are, at all times, a known technical variable. Using information about the locations and movements of all targeted objects both as operational elements of the network and as the basis for further calculations responds to the cybernetic imagination. It results in a comprehensively controlled world - a dystopian vision in which uncontrollable motion is no longer possible because relativity seems obsolete. But relativity can never be overcome, so perhaps the impossibility of its fulfillment carries a potential for resistance. Even if the desire for overcoming relativity is fulfilled, it is never fulfilled enough to dissolve. The solution for a desire remains bound to the imaginary of its initiation.

Although mobile technologies have become dominant cultural influences and mobility studies emerged in the past decade to explore the manifold practices surrounding it (e.g. Goggin, 2012), it is surprising to see that the technological dimension of cellular triangulation has rarely become the topic of theoretical or historical studies (for an exception, see Schäffner, 2011). In the first step of this article, Agre's still highly topical concept is applied to today's mobile media networks. In the second step, this theoretical approach is referred to the technologies of cellular triangulation, and in the third step to an example developed at the same time as Agre's text: ubiquitous computing at Xerox PARC and the spatial restructuring of local address space taking place in this context. This formation is finally connected back to the cybernetic imaginary in the fourth step, in which the implied ontology of addressability is explained. Finally, by going beyond Agre's notion, the last step presents different modes of interruption, of un-addressability, and of standstill not as a romantic opposition, but as both a theoretical and a technicalhistorical intervention into the cybernetic imaginary even if it seems to be realized.

\section{Capture and surveillance in mobile cellular networks}

The movements made by and with addressable devices in digital networks of mobility are not only the object of data collection; they generate the very data in question. From both a technological and a social perspective, then, mobility enabled by addressability is a constitutive element of these networks and permits extensive processes of data acquisition. These 
procedures are the basis for the economic exploitation of digital networks. As early as 1994, and thus before the advent of smartphones and social media, information scientist Philip Agre (1994) referred to this mode of data acquisition as the "capture" model. Agre's text draws a distinction between two modes of tracking and collecting data about human activity — the surveillance model and the capture model — in order to offer a clearer picture of organizational and work processes that were the topic of his studies. Whereas, in the case of the surveillance model, the activity to be observed and evaluated is accompanied by a second scopic act of monitoring (as in the video surveillance of people working on an assembly line), in the capture model these two activities collapse into one. Supported by technical systems of data acquisition, the individual activity produces the very data that are registered. This act of registration is an automatic component of the activity itself. In this way, activities are controlled and formatted in the act of data acquisition. By way of example, Agre mentions the monitoring of cargo trucks by means of GPS devices, electronic ankle bracelets, and tracking technologies that today form the basis of the Internet of things. Agre's text aims to classify these two modes within economic contexts in which, since the beginning of industrialization, various methods of data collection have been implemented. Capture and surveillance should therefore not be understood simply as opposites or as different stages of historical development. Agre's model stands apart by recognizing that the act of surveillance is not restricted to intelligence services, but is rather a starting point for methods of data collection implemented in our everyday lives, in urban cohabitation, and in production processes.

Agre shows that the standardization of work and organization processes via automatized formatting fosters the optimization and rationalization of such procedures. For Agre, capture is always an instance of economization in which repeatable and predictable forms and behaviors are extracted from the diversity of human activities. These forms are exchangeable with low transaction costs. The dimension of immaterial labor, the value generated from affective and cognitive activities relying, for example, on user-generated content, today closely bound to processes of capture in social networks or targeted advertising, was hardly conceivable at the time of publication. Nonetheless, the text is productive in application to these practices, as is obvious in its extension to the economies of platforms such as Facebook, Twitter, or Google (see Gerlitz and Paßmann, 2014).

The act of data collection in Agre's model implies translating collected data into a system for further processing. On the basis of this processing cycle, as Till Heilmann (2015) has stressed, capture "can be realized as a formal-linguistic representation of processes $[\ldots]$ by institutions with entirely different organizational structures and motivations" (p. 39). By means of so-called "grammars of action" (Agre, 1994: 107) — a term that was used at the time to describe organizational routines (see Pentland and Rueter, 1994) - the activities recorded automatically by the capture method are transferred into a catalog of prescribed schemata. They are standardized, on the basis of their linguistic classification, in such a way that they can be transformed into data sets amenable to further processing. Just as grammar defines what can be said, the grammar of action, as a quasi-linguistic set of rules, defines what can be done.

For Agre, the development of procedures of capture is part of a "reorganization of industrial work activities to allow computers to track them in real time" involving logistical methods for tracking the movement of products within distribution networks and for 
optimizing processes at the workplace (Agre, 1994: 101). Control in the capture mode is therefore closely associated with the capitalist logic of exploitation and the rise of neoliberalism. As Mark Andrejevic (2007) and Seb Franklin (2015) have pointed out, Agre's model provides a conceptual framework for describing late 20th-century production and consumption in terms of the collection and processing of data. Capture and surveillance are thus part of what Heilmann, inspired by Agre's approach, has called "capture capitalism." According to Heilmann, the latter is not oriented toward the contents of communication but rather toward the production of exploitable data. This sort of data collection is less concerned with messages than it is with information about movements, processes, and activities. Their analysis and further processing do not require an additional step but are rather an integral element of the organization of production and, increasingly, consumption as well.

Methodologically, Agre's text offers the opportunity to understand surveillance and capture in terms of the ways that they blend together the social and the technical. Following Agre, methods of documenting patterns of motion or work processes are neither purely technical mechanisms of data processing nor purely social processes of surveillance. The technical processes are rather an integral element of the social networks in which they operate. As Agre (1994) demonstrates, both modes of data collection are embedded in the larger context of a capitalist and neoliberal logic in which knowledge about the movements and locations of bodies forms the basis for controlling them: "By imposing a mathematically precise form upon previously informalized activities, capture standardizes those activities and their component elements and thereby prepares them [...] for an eventual transition to market-based relationships" (p. 120). In this sense, capture converts movements and activity into economic entities to be exploited in the form of data.

Whereas Heilmann discusses this model in terms of the history and logic of digital technologies and treats it as a capitalist strategy, here I would like to apply it to a different phenomenon: the collection of data about movements in space by means of mobile addressing. Although Agre mentions processes of this sort (he discusses RFID chips and barcodes), his examples involve networks with central receivers instead of networks of mobile participants. On one hand, the form of mobile addressing based on, for example, cellular triangulation makes it technologically possible to determine the location and movement of the addressed devices; on the other hand, it is based on technologies of capture, in which addressability generates information about the location of devices and fosters movement. In this regard, it is possible to understand capture processes as one of the central technical activities of the present - and thus as attempts to implement the cybernetic imagination.

\section{Cellular triangulation and the smartness of mobility}

In the following step, the model of capture is applied to technologies of cellular triangulation. To move through the world and its technical infrastructures with a cellular device is to be constantly reachable at the respective location where the device can be addressed. The technical challenge, to which cellular triangulation reacts, is the ability of networked objects to move while retaining their address in order to be constantly reachable. A 
smartphone possesses a multiplicity of addresses: the geographical coordinates on the surface of the earth (Global Positioning System), the assigned IP address (Internet Protocol), the device's individual MAC address (Media Access Control), the IMSI address (International Mobile Subscriber Identity) stored on its SIM card and needed to $\log$ in to the network, the device's unique IMEI address (International Mobile Equipment Identity), and finally the number with which it can be called (Mobile Subscriber Integrated Services Digital Network Number). As participants of mobile networks with the current standards GSM (Global System for Mobile Communications), UMTS (Universal Mobile Telecommunications System) and LTE (Long Term Evolution), devices require the last three addresses. ${ }^{1}$ By moving through the spaces covered by these networks, cellular devices produce data that are necessary to maintain a connection within the network and to authenticate and authorize themselves. In order to ensure the constant reachability of a phone even when it is moving around or changing base stations - in order to ensure, that is, the continuity of reception - the location of the device is determined by the act of addressing in relation to the surrounding cell towers. Although devices in mobile networks can also be stationary, constant addressability makes it possible to move through the geographical space covered by the network without losing contact.

The development of permanent addressability via cellular triangulation begins in the 1950s at Bell Labs and Motorola (Chapuis and Joel, 2003). Invented by Donald Ring in 1947, the cellular system is patented by Amos Edward Joel for Bell Labs in 1972 (Joel, 1972; Ring, 1947), commercially established for the first time in Tokyo by Nippon Telegraph and Telephone in 1979, and standardized on a global scale with the first generation G1 in the 1980s. ${ }^{2}$ In a network with only one strong base station, movements of senders and receivers lead to interferences in the spectrum of frequencies. For this reason, Ring suggests a cellular network no longer centered toward a single antenna. The solution realized by Joel consists in replacing a central antenna by a network of smaller transmitters. This mobile network is defined by a hexagonal structure of cells. Each cell consists of the area covered by three antennae on three different cell towers, each covering an angle of $120^{\circ}$. Every cell overlaps with the neighboring cells and uses a different frequency. Depending on local requirements and geographic, meteorological, and architectural factors, cell sizes can vary: from a radius of $35 \mathrm{~km}$ in rural areas to femtocells of a few meters inside of buildings or, for example, in the stores of providers.

In case of an incoming call for a mobile phone, the device is identified with the help of the phone number in the provider's permanent home location register. Addressing the correlated IMSI, the device's last check-in to a specific location area is then retrieved from a temporary visitor location register, which holds available location data as long as the device is logged in to a location area. A location area consists of a set of base stations and transmitters that are grouped together to optimize signaling. After the database query, the stations of this area send a paging signal to all devices in their area containing the IMSI as a header. If the addressed device is available, wireless connectivity is provided within fragments of seconds. The base station connects the device to the wired network of the provider.

Localization by reference to the selected location area supported by the visitor location register is only one possibility of positioning in the network. A second possibility 
becomes important when a device leaves the coverage of a cell during a call or data. In order to be portable without losing connection, a mobile phone has to be aware of its own location and be in constant communication with the surrounding cell towers for the socalled handover. ${ }^{3}$

To achieve this, all towers send out a signal via the broadcast control channel which is constantly monitored by the device. Depending on this signal, the device logs in with the strongest cell tower. By using so-called pings, signals are sent between towers and devices that request the receiver to send a return-signal, whose run-time makes it possible to calculate the distance from the respective tower. The calculations of the device are sent to the base station controller which decides upon possible handovers to other cells. Depending on the signal strength and the pings, the distance to a cell tower can be calculated, but not the exact location. The radius in which the device is positioned could be vast. But by counting in the localization of three cell towers and their overlap, the position of the device can be triangulated with an accuracy of a few meters, depending on the density of towers. If the device was connected to one central tower, it would only be possible to determine its position in the $120^{\circ}$ range of the station. Because mobile devices constantly measure the signal strength of all surrounding towers and switch to the strongest, network providers can determine every registered device with an accuracy of 2-5 m in urban areas. Mobile networks need this information to optimize reception and to define the base station sending data to a device.

In this sense, mobile networks employ capture processes as an integral part of the practices about which data are collected. Mobile communication networks can only operate because the devices constantly document their location and have addresses that move along with them. In order to be portable - that is, in order to be able to move from one cell tower's area of transmission to another's - a mobile phone has to be aware of its own location and be in constant communication with the surrounding transmitters: "It can always be located," as Wolfgang Hagen (2009) has stressed, "because it locates itself' (p. 367). Because there is a channel to it, every address authenticates the existence of its connectivity within a network. Addressed positions are no longer firmly linked to geographical coordinates but are rather just as mobile as the devices that codetermine their possible applications. Consequently, the last link between tower and device is where mobility happens. The rest of the network, the infrastructure of transmission, is immobile. Beyond the scope of these networks, there are no addresses and thus no way to locate devices.

It would be worthwhile to situate these technologies in the context of the long history of postal procedures of addressing that Bernhard Siegert (1999) has described in his book Relays. Mobile addressing continues some aspects of postal delivery, but differs in three important respects: first, the coupling of addressing and localizing with movements in space; second, the self-registration of this movement, in which data about the movement is collected in the act of motion itself; and, third, the communication between moving objects that this enables. Networked devices in cellular mobile networks are now linked to geographical space in a new way: They are addressable and they have to be locatable, but they can move. Whereas transmitting ships, satellite phones, or walkie-talkies likewise communicate while in motion, cellular devices go beyond this by registering their location by their very motion. ${ }^{4}$ The position of a cellular device can potentially be 
determined at any time and is known within operations of the mobile network. It is not necessarily stored (the withdrawn EU Data Retention Directive only attempted to regulate that information about the used cell should be retained), but is important for the functionality of the network. In this context, addressing and locating are not optional applications but are rather a technical precondition of the technologies in question.

\section{Ubiquitous computing and the tracking of movement}

Insofar as localization is inherent to the operations of such a network, questions about the spaces of these technologies arise: Their space of address becomes a technically pervasive environment created by the relations between addresses. So much was already evident in the first three devices that were developed under the name of "ubiquitous computing" (see Want, 2010 and Weiser, 1991). Although they are not related to cellular triangulation, these devices can be understood as a part of the prehistory of the smartphone and mobile connectivity; moreover, they demonstrate that technologies of addressing and their coupling with capture processes were central to the introduction of the first mobile digital systems. These technologies were attempts to ensure a state of reachability even under conditions of constant motion. Their example is invoked here as a case study not only because it is well documented, but also because in this context philosophical implications of these new technologies were discussed.

Ubiquitous computing - to offer a brief historical sketch — was conceived and developed in the early 1990s at Xerox's Palo Alto Research Center (PARC) in California (Dourish and Bell, 2011). The team behind it, which was led by the computer pioneer Mark Weiser, understood the term to denote a fundamental shift of computers away from desks and server rooms and into the all-encompassing background of everyday life. Motivated by the fact that components had become smaller and less expensive, portable handheld devices were supposed to merge with the environment by becoming ubiquitously mobile and invisible at the same time (Weiser, 1991). Many of today's most influential developments were not only anticipated at PARC but also patented, prototyped, networked by means of the lab's in-house protocols, and tested in offices and daily life. The researchers also thought through the basic ideas and concepts (including the philosophical and social implications) of a smart network of things. Despite the different technologies, the reference to PARC demonstrates how the spatial effects of the "next generation computing environment" (Weiser, 1993: 75) were negotiated. The mobility anticipated at PARC was based not on telephones, but on miniaturized computers.

From the beginning, the challenge of mobile data transmission was inscribed into the ambitions of ubiquitous computing. One of the central objectives of the project was to create environments which automatically adapt to the needs of the user, for example, by adjusting the appropriate temperature, or automatically forwarding phone calls upon entering a room. To achieve this, both continuous tracking and context-sensitive environmental awareness are necessary. The three most important prototypes developed at PARC and tested in offices during the early 1990s were the LiveBoard, which was a sort of digital blackboard; the ParcPad, which was the size of a book; and the palm-sized ParcTab. They were designed, respectively, to function as a display, a notebook, and a handheld device and their scale corresponded to a yard, a foot, and an inch (see Dourish, 


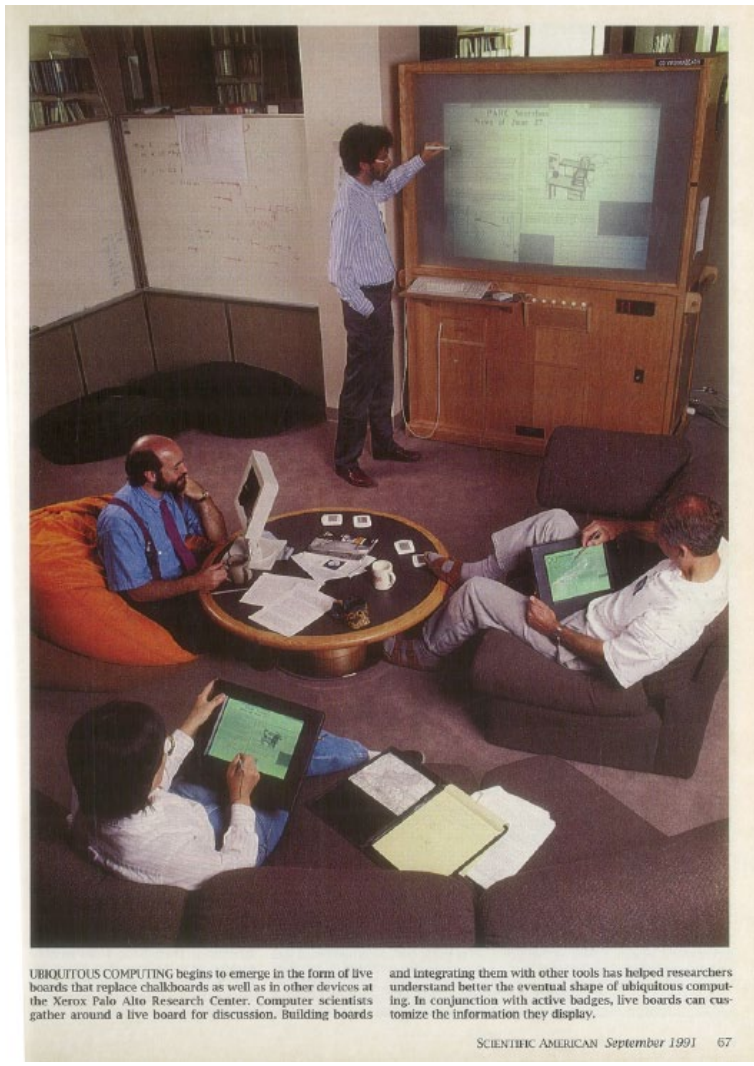

Figure I. Office meeting at Xerox PARC (Weiser, 1991).

2001)All three types of networked devices employed space-dividing functions. LiveBoards, which were fastened to walls, were meant to display identical content in different conference rooms and thus to enable meetings to take place with remote colleagues (see Figure 1). With LiveBoards, it was possible to teleconference and to work collectively on a single document from the different locations where identical content was being represented.

Whereas LiveBoards were immobile, ParcPads could be placed anywhere and did not have to be carried around at all times on one's body. Equipped with touchscreens, they were not personalized devices but could rather be accessed by different users by means of passwords. Any user could conceivably access his or her personal data on any Pad. Unlike today's smartphones and tablets, a ParcPad was not a hyper-personalized object. Once finished with it, a user would rather leave a Pad where it was, where it could then be picked up and used by the next person.

The ParcTabs, which were equipped with three buttons and a touchscreen and also existed in a simplified variant as a networked name tag, were identified with their users and served to locate them within the confines of the research institute. The Tabs were 


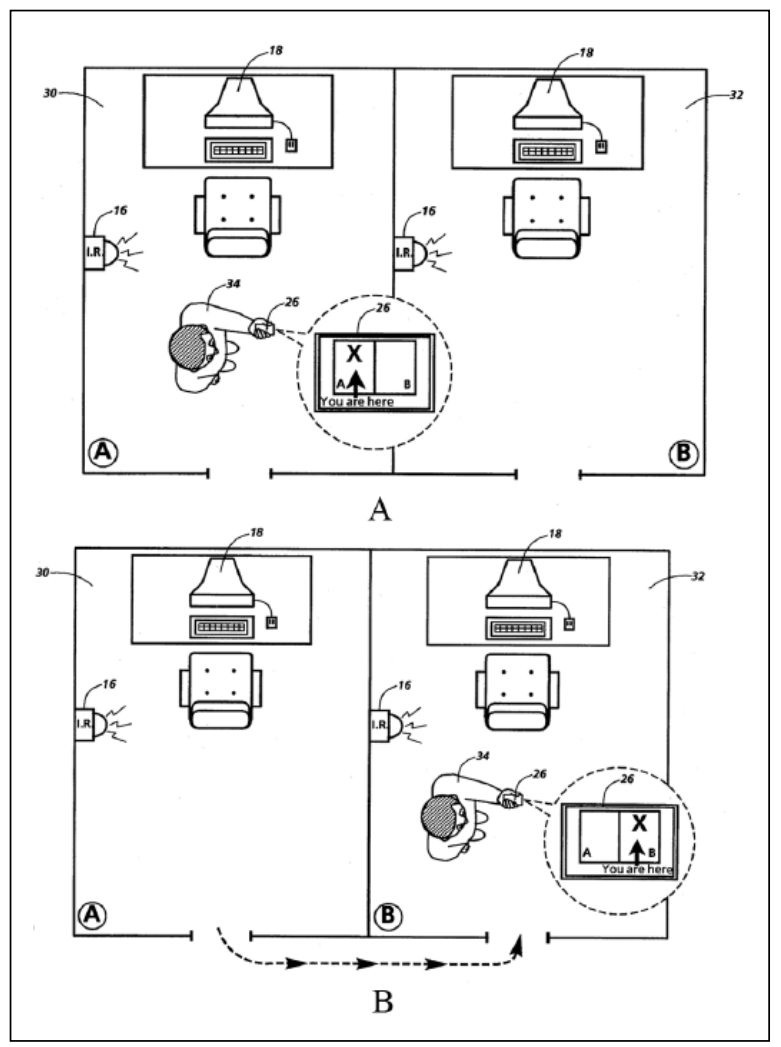

Figure 2. Example of ubiquitous computing environment (Want et al., 1993).

inspired by the so-called Active Badges that Roy Want, a member of Weiser's team, developed for Olivetti Research in the late 1980s. Every Tab had its own address that was associated in a database with the identity of its user. Initially, because of the prototypical application, there were only 20 Tabs in 25 cells (Want et al., 1995: 39). Coworkers were able to send messages to each other, exchange data, control the air conditioning, and play games. The Tabs also communicated independently with each other about the context and environment of their users' location in order to provide exact information about who is with whom, where network access is available, and if there are still unanswered emails on the stationary desktop.

In this early stage, packet-based signal transmission for Pads utilized license-free short-waves with rates of $250 \mathrm{kbps}$ and for Tabs energy saving infrared with $10 \mathrm{kbps}$. While Pads were used to display contents from the intranet, Tabs focused on locationbased services. Infrared-receivers with a range of 4-5 $\mathrm{m}$ were used as base stations with access to the local Ethernet and installed at the ceiling of each room. In 1993, this method was patented with the telling title "Method and System for Maintaining Processing Continuity to Mobile Computers in a Wireless Network" (Want et al., 1993). Infraredtransmission had a huge advantage over other technologies: Such signals cannot pervade 


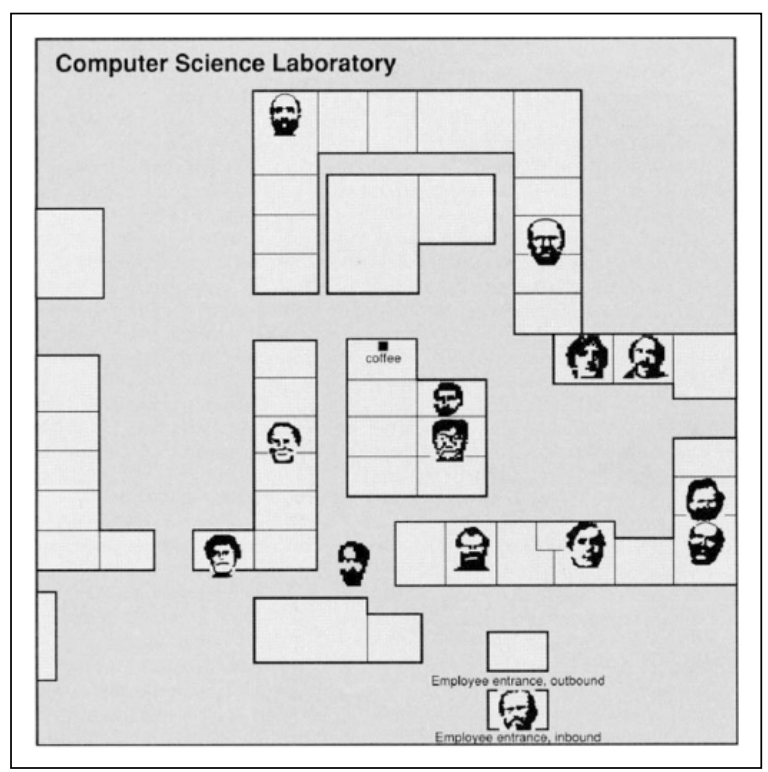

Figure 3. Map of PARC offices (Weiser, 1993: 8I).

walls. Consequently, every room could use the same frequency without taking the risk of interferences. To build a cellular infrared system was simple because if the device were connected to a receiver, it was necessarily located in the corresponding room (Figure 2[a] and 2(b)).

The process of locating the Tabs created a new correlation among the addressed. In constant intervals, the Tab sent an identifying signal, a beacon, to the available receiver to be located. On a map that could be displayed on the LiveBoards and Pads, the position of every Tab, visualized by the face of the corresponding user, within the building was shown (Figure 3). In practical terms, this knowledge of everyone's whereabouts, limited by the architecture of the PARC building, made it easy to forward telephone calls to the nearest phone, hold the elevator automatically at the appropriate floor, or see who is still interested in watching the beginning of a football game (Want et al., 1995: 29).

In these three devices, it is possible to distinguish three forms of built-in mobility that served what Nigel Thrift and Shaun French (2002) have called the "automatic production of space": meeting and broadcasting meetings for multiple users gathered in front of one device; providing local and networked Pads for anyone to use within a given space; and locating people with Tabs, which allowed individuals to be organized. Functionally, these three levels were closely connected and could be coordinated, by means of inhouse protocols, in such a way that the employees at PARC could — in the ideal situation (these were still prototypes, after all) — move seamlessly between their different applications and between floors and rooms without losing connectivity. They thus formed an environment that was not defined by a central location and did not simply correspond to a system of Cartesian coordinates made up of user-friendly representations on a screen 
(Figure 3). While Pads and LiveBoards were able to visualize positions, the Tabs created an environment of mutual reference: though due to lack of a display, they were not able to present maps, they registered each other and coordinated cooperative work. Out of the collected data, a relational address space was calculated that mapped out the position of every object and their relation to one another. With these relations between addresses, it was possible to determine positions. What was thereby implemented was a connectivity of things that were linked to one another, operated reciprocally as transmitters and receivers, and integrated their functions into a continuum of applications. Already at this early stage of ubiquitous computing, the technically implemented environment was not identical to the prescribed geographical space but was rather an effect of mobile addressability.

\section{The ontology of addressability}

This transformation of surrounding environments by mobile technologies, whose early parameters can be observed at PARC, is accompanied by the necessity of redefining spaces of addressing and positioning in which objects continuously receive assigned addresses not only during transmissions but are also located during movements within networks. What is novel about cellular networks (also compared with the technologies used at PARC) is that the objects themselves all function as agents of mediation, because they constitute an environment in which every other object's position is registered relationally in the act of movement and not in reference to geographical coordinates. Positions in mobile media networks are positions in relation to other devices that consequently act as mediators.

In this regard, the development of ubiquitous computing at PARC already amounts to a constant localization of all participants and their spatial relations. The location of every networked object within this relational space is necessarily known and can be visualized in an early version of a location-based service (Figure 3). In this context, unambiguous addressing by means of capture allows for spatial and temporal tracking, an index of traversed paths, and thus the ability to collect data, which can potentially be used to optimize processes or to create profiles. If movement data are processed and analyzed, it is possible to draw conclusions about patterns of the movement of employees, their routines, and involvement into teamwork.

Beyond making it possible to locate things and people geographically, addresses contribute to numerous social practices (by regulating who can address whom and who cannot) and also make it possible to register this very activity: in the context of big data, addresses allow prior actions to be analyzed in order to make predictions about future movements and to analyze graphs of social relations. Finally, computerassisted addressing processes are also used to decide - with or without the knowledge of the addressed - who is and who is not permitted to be present at a given place and time.

However, compared to the operations of a mobile network, the storage and processing of movement data is a retroactive step. As Oliver Leistert (2013) argues, the utilization of such data transforms operational data inherent to the running of a network into second-order information: 
The retained data has no computational function anymore, but it is transformed into the realm of the symbolic: It now represents the movements and telecommunication acts of people, whereas previously, it was not placed in the register of representation at all. (p. 158)

The potential utilization of movement data - especially in combination with metadata about communication behavior and in reference to addressees - poses a variety of juridical, political, and ethical questions (e.g. Well, 2014). Nonetheless, the ephemeral operational data of addressing are important on a different level and require a media-archeological approach. They are immanent to mobile networks. They facilitate movement within networks and thus generate not only a specific space, but also an ontology of what exists in the network and what does not exist. Thus, temporary operational data, in contrast to second-order information derived through the analysis of stored data, involve politics on a very different level regarding the technological implications of operational modes.

The spaces in which mobile networked devices are able to move around can be understood as surrounding spaces, as calculated and calculating environments. In these environments, as in all digital networks, every object has an unambiguous address with which it can be located. But in cellular networks, the virtual topology of address spaces is created by constant registration of movements that can then be projected onto the geographical spaces in which devices are able to move. In this movement, positional data are acquired that in turn constitute this environment. Because the relational environment of connected devices fosters mobility by capturing data, it cannot simply correspond to geographical space. The net is not the territory. This environmental topology of mobility in which movement and data about this movement converge needs to be taken into account in order to understand what is at stake with these technologies and their ontology of addressability. Every object that is networked in this way has to monitor its connection to base stations on an ongoing basis in order to maintain the functionality of the network. On one hand, the environment of cellular triangulation is calculating insofar as the position of every device is constantly tracked for it to remain a part of the network. On the other hand, the environment is calculated because addressing is immanent and it only exists if the positions of all connected devices are registered. In the capture mode, the calculation of fluctuating spatial relations takes place by means of the movement of the devices themselves. To call the space spanned by these technologies an environment (rather than simply a map of geographical locations) recognizes that it is created through the relations of surrounding between devices and base stations.

The space occupied by these objects, whose givenness consists in their connectedness, is constituted relationally through the captured information about the position of its elements. Regardless of the limited range of certain infrastructures, objects that are assigned addresses are linked to an environment whose interior is not opposed by an exterior and which is constituted by capturing the addresses of the objects within it. As a space for the distribution of data and arrangement of objects, this technically permeated environment is ubiquitous in the sense that addresses are no longer tied to stationary geographical locations but are rather able to move around. However, this relational space of mobility, in which positions can only be reached if they can be addressed, is limited by the strength and range of signals and by the ends of its channels. Movement is always performed in relation to infrastructures. Within these limits, the scope of the network is 
defined by the correlations that exist between distributed addresses, as Jordan Crandall (2010) has argued: "[Calculative mobilization] generates an 'enhanced' environment in which potentially every entity, defined in terms of its location and its tracked and anticipated movements, can become the subject of its calculative procedures" (p. 76). Outside of this environment, there is no place for networked objects. They may move, but they cannot move out of the network without losing their status as networked objects. What is not networked and thus not addressable cannot be part of the environment. Addressing all objects is potentially associated with an ontology according to which only that exists which has an address and is networked. Access to the network is bound to the condition of addressability. This ontology only knows two states: existence, which means addressability, and non-existence, which means having no address. Even being temporarily unavailable means having an address - and in mobile networks the addresses can move constantly without losing connection. With mobile devices, the virtual topology of address spaces becomes a relational environment because the data about addresses are captured in acts of movement through space. In this regard, existence, as connectivity, is an infrastructural variable. Despite the density of its addresses, the space of calculations is not ubiquitous but rather tied to specific infrastructures. It can only extend as far as its channels, which consist of cables and plugs, cell towers and devices, and which are useless without data centers and power supplies - components of mobile networks that are immobile.

\section{Conclusion: the imaginary of reachability}

In light of the processes of capture, addresses can be regarded as basic operators of mobility in digital cultures. The cybernetic imagination of overcoming the relativity of control is carried forward by technologies of capture that inextricably bind objects and their movements to the allocation of addresses. In this regard, cellular triangulation has emerged as one of the most sophisticated solutions to the challenge of relativity: that which is part of the network has an address; that which has an address has a registered position; if the position of an object is registered, it can move without losing connectivity. In this sense, addresses are elementary technical components of a society whose coherence is achieved, to an ever-growing extent, by means of transmissions - a society that increasingly depends upon digital networks. If technical and social networks converge-if the technical possibilities of connecting are superimposed onto existing social relations and new social relations are formed by means of technical channels, as can be seen in today's social media as well as in daily practices with mobile media - then social relationships can hardly be conceived any longer without recourse to technical networks of communication and their infrastructures. The ontology of addressability, concerning all technical networks, has become a political requirement of participation. But in the case of mobile networks, addressability also implies traceability and trackability. The consequences of this development are at times contradictory - as all technologies, cellular triangulation is a pharmakon, a remedy and a poison at the same time (Derrida, 1981). Nonetheless, at this point it is possible to draw a handful of conclusions and to at least give a hint at the potentials of un-addressability. 
If the networks of mobile media enable not only the simultaneous reachability and movement of their participants, but are in fact created by addressing, which in turn makes movement possible, what does this then mean for Tiqqun's observation, cited at the beginning, that the history of cybernetics consists in attempts to discover technical processes for simultaneously determining the location and movement of objects? Simultaneously determining the location and movement - the position and behavior - of an object is physically impossible because, as the example of anti-aircraft predictor makes clear, the target will have changed its location by the time that information is received about its location. By means of feedback circuits, early cybernetics made successful attempts to overcome this problem at least by approximation. The possibilities of mobile addressing are part of the new solution strategy. In these networks, addressing, which means the relational determination of a position, is a precondition of movement and of changing the position while retaining the address. Position and movement turn into variables of one singular act of calculation. Actually, with the run-time of pings, relativity becomes a component of localization. The impossibility of instantaneous transmissions remains effective, but self-addressing objects localize their own position while moving and can only be part of a network because movement (between cells) and position (in reference to cells) are constantly registered through the triangulation of at least three surrounding cell towers.

The utilization of movement data as second-order information, as it is discussed in the context of privacy and surveillance, is a belated act in which movement and position can be correlated only after the act, but not simultaneously. In a strict sense, the utilization of movement data, for whatever purposes, is not a solution strategy because it does not touch the problem of simultaneity. Technically, it is situated on a different, secondary level compared to the operational data of addressing that facilitate the activity of the network. In processes of capture that are embedded into the operations of the network, addresses are elementary factors. They can be extracted and exploited, for example, in graph or big data analysis only retroactively. Mobile cellular networks would be inoperable without localization. It is inherent to the act of addressing, and this again is implemented into the operations of the network which afford movements.

On this operational level, technologies of capture allow targeted objects to record the traces of their own movement and thus, allowing for unavoidable transmission times, ensure that their location can be constantly determined. If, with Tiqqun, the history of cybernetics is a history of attempts to overcome the impossibility of simultaneously determining position and behavior of an object, then this history might take a new turn with technologies of capture. The dystopia seems to be realized. With our cellular devices in hand, we move around like enemy fighter jets that are always documenting where they are as they travel through an environment in which every position is identifiable. Only the infrastructures of addressability are not yet ubiquitous and usually so imperfect that constant failures to connect and dead spots affect their use. Often, reachability is prohibited by infrastructures, even though the body carrying the addressed device moves.

This relation of a receiving device to the body carrying it is arbitrary. The object of capture is always the device being addressed. Its connection to the human body whose profile is being analyzed and monetized, necessarily remains ambiguous. The movement of the body does not have to correspond to the movement of the device. While 
it is possible to concur with Tiqqun and understand the history of cybernetic control technologies as an attempt to overcome this uncertainty and to minimize contingency by correlating movement data with individuals, this relation can never be unequivocal. Although capture prestructurizes activities, they are nonetheless arbitrary and non-determined. Operational movement data therefore contain a certain potential for resistance through non-addressability — not in the sense of leaving the network but rather by appropriating the relation between body and device. ${ }^{5}$

This potential is important for those who are not (yet) part of these networks. Huge parts of the world are not connected and thus excluded from the ontology of addressability. In contrast, for those who are connected, this ontology has achieved an existential dimension. To be connected has become a criterion of existence. But which options are open inside of this ontology? Which potentials of resistance can be imagined that do not lead to the simple rejection of these technologies? How can they be undermined from the inside?

At stake are modes of non-addressability in which a device is part of a network without having its geographical position - and thus the supposed position of the user's body - registered. ${ }^{6}$ Numerous techniques of obfuscation have been developed with the aim of making addresses (and not just content) unrecognizable, from the metal strips (chaff) deployed by military airplanes to be invisible to radar and missiles, to the TOR-browser which enables anonymous communication by making it impossible to retrace data because of the hundredfold overlap of connections. As Finn Brunton and Helen Nissenbaum (2015) have demonstrated, these techniques and practices are meant to restore symmetry to potentially monitored communication. They are embedded within infrastructures, which means, they correspond to the technological architecture and to the protocols of the respective network. In a similar way, the arbitrary relation of body and movement can be used to enable non-addressability in cellular networks, for example, when devices are swapped between users, several devices use the same address, or mesh and peer-to-peer-networks render direct connections possible (see Leistert, 2013). ${ }^{7}$ In this sense, non-addressability could be transformed into an alternative to the binary ontology of addressability, in which a mode of existence in un-addressability would become feasible. It is specific for the dominant logic that non-addressability equals the nonexistence of an address, while bodies do not have addresses. The question remains, if there are modes of connection to the network in which bodies can become non-addressable but existing parts of the network.

In a world in which both location and movement are known and in which, at the same time, the phantasms of both mobility and constant reachability have become technical and social imperatives, it is also possible to stand still and refuse to leave one's place. In this sense, Darin Barney (2015) has pleaded for a "politics of immobility" and has identified, in the subversive act of willful paralysis, another resistant potential for undermining the constant movement of goods, capital, and people. Thus it is possible, he argues, to oppose mobility - in which location and movement are not only known but also its very condition - with an alternative. This consists neither in a radical act of staying put, as Paul Virilio has described, nor in the phantasm of deceleration proposed by Hartmut Rosa. Such approaches to withdrawing from movement remain mired in the dispositif of 
mobility itself. Much like Tiqqun, Barney rather suggests that we direct our attention to the infrastructures of mobility - to that which correlates positions with movements. Infrastructures are those entities that stand still amid all the crisscrossing motion around them, but they do not belong to the people who use them. There can be no movement without infrastructures. They control the time in which movement takes place if it is a part of the network. Infrastructures make movements probable. Immobility, as the free movement of a body, is therefore the maximum contingency in a system designed to minimize contingency.

\section{Acknowledgements}

Earlier presentations of this paper were presented on different occasions at Universiteit Leiden, King's College London and the University of Siegen. The author is very grateful to Pepita Hesselberth (Leiden), Bernard Geoghegan and Jonathan Gray (London), Marcus Burkhardt, Katja Grashöfer, Mary Shnayien, and Bianca Westermann (Siegen) for the chance to present and discuss his ideas. He would also like to thank the two anonymous reviewers for their helpful and, in the best sense of the word, challenging reviews.

\section{Funding}

The author(s) received no financial support for the research, authorship, and/or publication of this article.

\section{Notes}

1. For the sake of brevity, the following explanations refer to Global System for Mobile Communications (GSM), which was introduced in 1990. Although structurally similar, Universal Mobile Telecommunications System (UMTS) and Long Term Evolution (LTE), which use packet-based transmission and consequently made mobile Internet access feasible, differ in several aspects (Wakefield, 2007).

2. The historical development of mobile networks is closely bound to attempts to connect cars to local radio networks and to build car telephones (Buschauer, 2014: 415f).

3. A third possibility is offered by Apple and Google for iOS and Android: through databases of coordinates of huge numbers of WiFi-networks, devices can easily and quickly localize themselves.

4. GPS technologies, which use the distance to satellites to localize themselves, differ from cellular triangulation, because GPS devices do not form a network. They use signals sent from satellites to determine their geographical coordinates, but cannot communicate or be tracked. GPS is no technology of capture (on geolocation see Barreneche, 2012).

5. As Pepita Hesselberth (2017) has described, discourses of disconnection remain bound to conflicting assumptions about what it means to withdraw from a network (see also Bollmer, 2016).

6. Tellingly, in the lawsuit against Tarnac 9, a group connected to Tiqqun, which ended with an acquittal in 2009, one of the main charges was that the group did not use cell phones (Mandraud and Monnot, 2008). Philip Agre was reported missing in 2009. Although he was found by the police, he supposedly wants to live off the grid and never returned to the university (Carvin, 2010).

7. An example for an alternative model is the mesh-network Briar that enables peer-to-peer messaging without Internet access. See https://briarproject.org/ 


\section{References}

Agre PE (1994) Surveillance and capture: two models of privacy. The Information Society: An International Journal 10(2): 101-127.

Andrejevic M (2007) Surveillance in the digital enclosure. The Communication Review 10: 295317.

Barney D (2015) We shall not be moved: on the politics of immobility. In: Herman A, Hadlaw J, and Swiss T (eds) Theories of the Mobile Internet: Materialities and Imaginaries. New York: Routledge, pp. 15-24.

Barreneche C (2012) Governing the geocoded world: environmentality and the politics of location platforms. Convergence: 18(3): 331-351.

Bollmer G (2016) Inhuman Networks: Social Media and the Archaeology of Connection. New York: Bloomsburg Publishing.

Brunton F and Nissenbaum HF (2015) Obfuscation: A User's Guide for Privacy and Protest. Cambridge, MA: The MIT Press.

Buschauer R (2014) (Very) nervous systems: big mobile data. In: Reichert R (ed.) Big Data: Analysen zum digitalen Wandel Von Wissen, Macht und Ökonomie. Bielefeld: Transcript, pp. $405-436$.

Carvin A (2010) Missing internet pioneer Phil Agre is found alive. Available at: https://www. npr.org/sections/alltechconsidered/2010/01/missing_internet_pioneer_phil.html (accessed 7 March 2018).

Chapuis RJ and Joel AE (2003) 100 Years of Telephone Switching: Electronics, Computers and Telephone Switching. Amsterdam: IOS Press.

Crandall J (2010) The geospatialization of calculative operations: tracking, sensing and megacities. Theory, Culture \& Society 27(6): 68-90.

Derrida J (1981) Plato's pharmacy. In: Derrida J (ed.) Dissemination (trans. B Johnson). Chicago, IL: The University of Chicago Press, pp. 63-171.

Dourish P (2001) Where the Action Is: The Foundations of Embodied Interaction. Cambridge, MA: The MIT Press.

Dourish P and Bell G (2011) Divining a Digital Future: Mess and Mythology in Ubiquitous Computing. Cambridge, MA: The MIT Press.

Franklin S (2015) Control: Digitality as Cultural Logic. Cambridge, MA: The MIT Press.

Galison P (1994) The ontology of the enemy: Norbert Wiener and the cybernetic vision. Critical Inquiry 21(1): 228-266.

Gerlitz C and Paßmann J (2014), Good' platform-political reasons for ,bad' platform-data. Zur sozio-technischen Geschichte der Plattformaktivitäten. Mediale Kontrolle Unter Beobachtung 3(1): $1-40$.

Goggin G (2012) Encoding place. In: Wilken R and Goggin G (eds) Mobile Technology and Place. New York: Routledge, pp. 198-212.

Hagen W (2009) Zellular—Parasozial—Ordal: Skizzen zu einer Medienarchäologie des Handys. In: Döring J and Thielmann T (eds) Mediengeographie: Theorie-Analyse-Diskussion. Bielefeld: Transcript, pp. 359-380.

Heilmann TA(2015)Datenarbeitim “Capture”-Kapitalismus:Zur Ausweitung der Verwertungszone im Zeitalter informatischer Überwachung. Zeitschrift für Medienwissenschaft (2): 35-47.

Hesselberth P (2017) Discourses on disconnectivity and the right to disconnect. New Media \& Society 20(5): 1994-2010.

Joel AE (1972) Mobile communication system. Patent.3,663,762. 16 May. US.

Leistert O (2013) From Protest to Surveillance: The Political Rationality of Mobile Media: Modalities of Neoliberalism. Frankfurt: Peter Lang. 
Mandraud I and Monnot C (2008) Les neuf de Tarnac. Le Monde diplomatique, 20 November. Available at: https:/www.lemonde.fr/societe/article/2008/11/20/sabotages-a-la-sncf-lesneuf-de-tarnac_1120974_3224.html

Pentland BT and Rueter HH (1994) Organizational routines as grammars of action. Administrative Science Quarterly 39(3): 484-510.

Ring D (1947) Mobile Telephony: Wide Area Coverage. Technical Memorandum for Bell Telephone Laboratories.

Schäffner W (2011) The telephonic revolution of the digital image. Grey Room 43: 144-155.

Siegert B (1999) Relays: Literature as an Epoch of the Postal System. Stanford, CA: Stanford University Press.

Thrift N and French S (2002) The automatic production of space. Transactions of the Institute of British Geographers 27(3): 303-335.

Tiqqun (2010 [2001]) The cybernetic hypothesis. Available at: https://theanarchistlibrary.org/ library/tiqqun-the-cybernetic-hypothesis (accessed 7 March 2018).

Wakefield T (2007) Introduction to Mobile Communications: Technology, Services, Markets. Boca Raton, FL: Auerbach Publications.

Want R (2010) An introduction to ubiquitous computing. In: Krumm J (ed.) Ubiquitous Computing Fundamentals. Boca Raton, FL: Chapman \& Hall, pp. 1-35.

Want R, Adams NI, Goldstein RJ, et al. (1993) Method and system for maintaining processing continuity to mobile computers in a wireless network. Patent 5,564,070. 30 July. US.

Want R, Schilit BN, Adams N, et al. (1995) An overview of the PARCTAB ubiquitous computing experiment. IEEE Personal Communications 2(6): 28-43.

Weiser M (1991) The computer for the 21st century. Scientific American 265: 94-104.

Weiser M (1993) Some computer science issues in ubiquitous computing. Communications of the ACM 36(7): 75-84.

Well A (2014) Ping! The admissibility of cellular records to track criminal defendants. St. Louis University Public Law Review 33(2): 487-518.

\section{Author biography}

Florian Sprenger is a professor for media and cultural studies at Goethe University Frankfurt. From 2012 to 2015 he was post-doctoral researcher at the Digital Cultures Research Lab at Leuphana University Lüneburg. His research focuses on the history of artificial environments, digital cultures and media epistemologies. 